L. de Montjoye ${ }^{1,2^{*}}$, A-S. Darrigade ${ }^{1 *}$, A. Giménez-Arnau ${ }^{3}$, A. Herman ${ }^{1,2}$,

L. DUMOUTIER ${ }^{4 *}$, M. BAECK ${ }^{1,2^{* *}}$

\title{
Correlations between disease activity, autoimmunity and biological parameters in patients with chronic spontaneous urticaria
}

${ }^{1}$ Department of Dermatology, Cliniques universitaires Saint-Luc, Université catholique de Louvain, Brussels, Belgium ${ }^{2}$ Institute of Experimental and Clinical Research, Pneumology, ENT and Dermatology Pole, Université catholique de Louvain, Brussels, Belgium ${ }^{3}$ Department of Dermatology, Hospital del Mar, Institut Mar d'Investigacions Mèdiques (IMIM), Barcelona, Spain ${ }^{4}$ de Duve Institute, Université catholique de Louvain, Brussels, Belgium

${ }^{* * *}$ These authors contributed equally to this work and should be considered as co-first/co-last authors

\section{KEY WORDS}

Chronic spontaneous urticaria; blood basophil count; IgE; autoimmunity; autologous serum skin test.

\section{Corresponding author}

Laurence de Montjoye

Department of Dermatology

Cliniques universitaires Saint-Luc

Avenue Hippocrate 10

B-1200 Brussels

E-mail: laurence.demontjoye@uclouvain.be

Doi

10.23822/EurAnnACI.1764-1489.132

\author{
Abbreviations \\ AAbs: autoantibodies \\ ANA: anti-nuclear antibodies \\ ASST: autologous serum skin test \\ BAT: basophil activation test \\ BHRA: basophil histamine release assays \\ CBC: complete blood count \\ CRP: C-reactive protein \\ CSU: chronic spontaneous urticaria \\ FceRI: high-affinity IgE receptor \\ IgE: immunoglobuline $\mathrm{E}$ \\ RF: rheumatoid factor \\ Tg: thyroglobuline \\ TPO: thyroperoxydase \\ UAS7: Weekly Urticaria Activity Score
}

\begin{abstract}
Summary
Background. Biomarkers of disease activity/severity and criteria of autoimmune chronic spontaneous urticaria (CSU) are still a matter of debate. Objective. To investigate possible correlations between clinical and biological markers and their associations with: 1) disease activity, 2) resistance to $H_{-}$-antihistamines, 3) autoimmunity and 4) autologous serum skin test (ASST) in patients with CSU. To also analyze biological parameter modifications in patients with CSU treated with omalizumab. Materials and methods. Disease activity, $H_{1}$-antihistamines response and presence of concomitant autoimmune disease were prospectively recorded in 95 patients with CSU. For 60 of them, ASST was performed. Broad biological analysis were performed. Results. C-reactive protein (CRP) serum levels were higher in $H_{-}$-antihistamines unresponders $(p<0.0001)$ and in more active diseases $(p=$ 0.033). D-dimer plasma levels were higher in $H_{1}$-antihistamines unresponders $(p=0.008)$ and in patients with autoimmune status (concomitant autoimmune disease andlor with autoantibodies) $(p=0.016)$. Total immunoglobuline $E(I g E)$ serum level was lower in patients with positive ASST. Blood basophil counts were lower in patients with CSU and especially in $H_{1}$-antihistamines unresponders $(p=0.023)$, in patients with more active disease ( $p=$ $0.023)$, with positive ASST $(p=0.001)$, and with autoimmune status $(p=0.057)$. Conversely, under omalizumab, a decrease of $C R P(p=0.0038)$ and $D$-dimer serum/plasma levels $(p=0.0002)$ and an increase of blood basophil counts $(p=0.0023)$ and total IgE serum levels $(p=0.0007)$ were observed. Conclusions. This study brings additional evidences of interest to investigate IgE, D-dimer serum/plasma levels and basophil blood counts in patients with CSU as they could be correlated to disease activity, response to treatment and/or autoimmunity.
\end{abstract}




\section{Introduction}

Chronic spontaneous urticaria (CSU) is defined as the spontaneous occurrence of wheals and/or angioedema daily or almost daily for more than 6 weeks. The pathogenesis of CSU has not been fully established although it seems clear that different mechanisms are involved. Mast cells have long been the key cells involved in CSU pathogenesis, however new evidence argues in favor of the involvement of other cells, i.e., basophils, eosinophils, lymphocytes, and neutrophils, as well as the involvement of cytokines, coagulation pathways and autoantibodies (AAbs). Autoimmune diseases, particularly autoimmune thyroiditis and thyroid AAbs, seem more prevalent in patients with CSU (1, 2). Several AAbs have been associated with CSU: IgG against thyroperoxydase (TPO) or thyroglobuline $(\mathrm{Tg})(1,3), \operatorname{IgG}$ against $\operatorname{IgE}$ or against high-affinity $\operatorname{IgE}$ receptor (FceRI) $(4,5)$, and $\mathrm{IgE}$ directed against autoantigens, such as TPO or interleukin $24(6,7)$. Furthermore, some patients with CSU react to the intradermal injection of their own serum resulting in a positive autologous serum skin test (ASST) (8). Also in vitro tests (basophil histamine release assays (BHRA) and basophil activation test (BAT)) showed that some CSU serum factors are able to induce histamine release/basophil activation $(5,9)$. The above-mentioned factors, alone or combined, are often used to classify patients as autoimmune or non-autoimmune CSU.

In this prospective cohort of patients with CSU, we analyzed correlations between several clinical and biological markers and their associations with: 1 ) disease activity, 2) response to $\mathrm{H}_{1}$-antihistamines, 3) autoimmunity, 4) ASST. We also analyzed biological parameter modifications in patients with CSU treated with omalizumab (anti-IgE treatment).

\section{Materials and methods}

This prospective study was conducted from September 2013 to December 2018 in the department of Dermatology of the Cliniques universitaires Saint-Luc, in Brussels, Belgium. The study and data collection were conducted with the approval of the institutional ethical committee. Informed consent was obtained from all patients.

\section{Patient selection and clinical data collection}

Ninety-five adults and adolescents ( $\geq 12$ years old) with a diagnosis of CSU, confirmed by a dermatologist according to the international Guideline 2013 (10), were included. Only patients with active CSU were selected. Patients with pure chronic inducible urticaria or bradykinin-mediated angioedema were not included in the study. Upon enrolment, medical history, including history of personal or familial atopy (asthma, atopic dermatitis and allergic rhinitis) and autoimmune diseases, as well as previous and current treatments for CSU were recorded.
Using a validated tool, the Weekly Urticaria Activity Score (UAS7), assessed disease activity (10). Patients were asked to record their symptoms for seven consecutive days prior to day of inclusion. Patients were classified as follows: severe CSU (UAS7 = 28-42), moderate CSU (UAS7 = 16-27), mild CSU (UAS7 = 7-15), well-controlled CSU (UAS7 = 1-6) and itch-and whealsfree (UAS7 = 0) (11).

Response to $\mathrm{H}_{1}$-antihistamines was also evaluated and assessed using the UAS7 over several months. Patients were classified as follows: $\mathrm{H}_{1}$-antihistamines responders (UAS7 $\leq 7$ with 1 to 4 tablets daily of $\mathrm{H}_{1}$-antihistamines); $\mathrm{H}_{1}$-antihistamines unresponders (UAS7 > 7 with 4 tablets daily of $\mathrm{H}_{1}$-antihistamines). Disease duration was defined as the time from the first onset of symptoms to day of inclusion. Recurring episodes of CSU, defined as recurrence of symptoms after at least 6 months of spontaneous remission, were also recorded.

For ASST and blood analyses, patients were considered as untreated, if they had stopped $\mathrm{H}_{1}$-antihistamines for at least 48 hours (or longer, depending on drug activity of each molecule) (8), anti-leukotrienes and $\mathrm{H}_{2}$-antihistamines for at least 7 days, and corticosteroids or cyclosporine $A$ for at least 1 month and have never taken omalizumab before inclusion.

\section{Autoimmunity and autologous serum skin test}

Patients were also classified according to their "autoimmune status". Autoimmune status was inferred in the case of a personal history of concomitant autoimmune disease or in the presence of at least one type of AAbs (included IgG against Tg and TPO, anti-nuclear antibodies (ANA) and rheumatoid factor (RF)). No autoimmunity was defined as the absence of concomitant autoimmune disease and AAbs.

ASST was performed on 60 untreated patients by the intradermal injection of $50 \mu \mathrm{L}$ of the patient's own serum into the volar part of the forearm (8). Prick tests with histamine and intradermal injection of normal saline solution served as respectively positive and negative controls. A positive test was defined as the appearance, within 30 minutes, of a red wheal with a diameter of $1.5 \mathrm{~mm}$ or greater than the wheal produced by the injection of normal saline solution. Patients were classified as either having a positive or a negative ASST.

\section{Biological tests}

Blood analyses include: complete blood count (CBC) with differential, platelet parameters, total IgE serum levels, thyroid function tests, serum levels of IgG against $\mathrm{Tg}$ and TPO, ANA, $\mathrm{RF}, \mathrm{C}$-reactive protein (CRP) serum levels, complement components (C3, C4), C1-inhibitor, classical complement pathways, protein electrophoresis, and D-dimer plasma levels. Titers were considered positive if IgG anti-Tg > $115 \mathrm{U} / \mathrm{ml}, \mathrm{IgG}$ anti-TPO $>34 \mathrm{U} / \mathrm{ml}$, ANA > 1:160, and RF > 1:40. For basophil blood counts, the reference range often used is from 0 to 200 or 300/ 
$\mu \mathrm{L}$. As this range is very large and start from zero, we used a reference mean for blood basophil counts which was established by the department of Clinical Biology of the Cliniques universitaires Saint-Luc based on healthy controls values.

\section{Omalizumab treatment}

For 22 patients treated with omalizumab (Xolair, Novartis, Camberley, UK) at the initial recommended dose of $300 \mathrm{mg}$ every 4 to 5 weeks (12), blood tests were performed before initiation and under omalizumab treatment.

Patients were classified according to their response to omalizum$\mathrm{ab}$ treatment as follows: complete responders if UAS7 was 0 , partial responders if UAS7 fell by at least 10 points (but UAS7 0 ), and non-responders if UAS7 remained unchanged, rose, or fell by less than 10 points. Based on time to response, patients were classified as early responders, if their UAS7 fell by at least 10 points after one month of treatment. Others were classified as late responders.

\section{Statistical analyses}

As some clinical or biological data may be missing for some patients, the number of patients studied for each parameter is always indicated. Data for categorical variables are expressed as frequencies followed in brackets by the number patients positive for this parameter over number of patients studied, and for continuous variables as mean \pm standard deviation (SD) with minimum and maximum values in brackets. The Pearson's $x^{2}$ test was applied to compare percentages of categorical variables. Mann-Whitney test and Kruskal-Wallis test were used to compare continuous variables between categorical variables. Wilcoxon matched-pairs signed rank test was used to compare paired variables, such as values before initiation and under omalizumab. The Pearson correlation coefficient was used to calculate correlation between continuous variables. In all tests, the level of significance was a two-sided $P$ value of less than 0.05 . All statistical analyses were performed, and graphs created using SPSS software Version 24 (SPSS, Chicago, IL, USA) and GraphPad Prism Version 8 (GraphPad Software Inc., USA).

\section{Results}

\section{Relevant patient data (table I)}

\section{Clinical data}

This study included 95 patients with CSU, 68 women (71.6\%) and 27 men (28.4\%). Mean age at inclusion was $45 \pm 16$ years and mean duration of CSU was $4.7 \pm 7$ years. Mean age of CSU onset was $40 \pm 16$ years. Angioedema was associated with wheals in 66 patients (69.5\%). Recurring episodes of CSU after at least 6 months of symptom free intervals without treatment were reported in $25.3 \%$ of patients (23/91). 58.9\% (56/95) were $\mathrm{H}_{1}$-antihistamines responders and $41.1 \%(39 / 95)$ were $\mathrm{H}_{1}$-antihistamines unresponders. Concerning disease activity based on UAS7, 24 patients (40.7\%) have severe disease, 10 patients (16.9\%) have moderate disease, 17 patients $(28.8 \%)$ have mild disease, 3 patients (5.1\%) have well-controlled disease and $5 \mathrm{pa-}$ tients $(8.5 \%)$ were itch-and wheals-free. ASST was performed on 60 patients and 24 (40\%) were positive.

\section{Biological parameters}

Biological tests that could be influenced by treatment (e.g., cell blood counts, platelet parameters, D-dimer plasma levels, IgE and CRP serum levels, complement components) were analysed only in untreated patients. Mean serum IgE levels was 208.2 $\pm 451.8 \mathrm{kU} / \mathrm{L}$, with $43.7 \%$ (31/71) of patients having levels higher than $150 \mathrm{kU} / \mathrm{L}$ and 23.9\% (17/71) having levels lower than $40 \mathrm{kU} / \mathrm{L}$. Mean D-dimer plasma levels was $1278.2 \pm 1939$ $\mathrm{ng} / \mathrm{ml}$, with $56.3 \%$ (40/71) of patients having levels higher than $500 \mathrm{ng} / \mathrm{ml}$. For both IgE and D-dimer levels, the mean values observed were higher than normal ranges and large variability was seen across patients. Blood basophil counts were lower in patients with CSU $(30.1 \pm 24 / \mu \mathrm{L})(74$ patients) compared with reference mean of healthy controls $(40.0 \pm 17.3 / \mu \mathrm{L})(\mathrm{p}=$ $0.019)$. No significant abnormalities were found in the rest of the $\mathrm{CBC}$, in protein electrophoresis, nor in complement.

Associations with autoimmune disease, autoimmune serology or atopy

One third of the patients $(32 / 94,34.0 \%)$ had clinical history and/or serological markers of autoimmunity, and therefore were considered as having a positive autoimmune status. Indeed, a concomitant autoimmune disease was present in 18.3\% (17/93) of patients, mainly thyroiditis (11/17), and AAbs were present in $30.5 \%$ (29/95), predominately anti-TPO (20/92). As well, familial history of autoimmune disease was found in $14,6 \%$ of cases (13/89). Nearly half of the patients (45/91, 49.5\%) had a personal history of atopy (based on anamnesis).

\section{Correlations between disease activity, response to $H_{1}$-antihistamines and clinical or biological parameters}

Response to $\mathrm{H}_{1}$-antihistamines was not correlated with clinical parameters such as angioedema, symptomatic dermographism, duration of the disease, age, weight, gender, personal or family history of atopy. In addition, no association was found between $\mathrm{H}_{1}$-antihistamines response and concomitant autoimmune disease, presence of AAbs, the positivity of ASST nor blood cell counts or total IgE serum levels. 
Table I - Clinical and biological data of the cohort of patients with CSU.

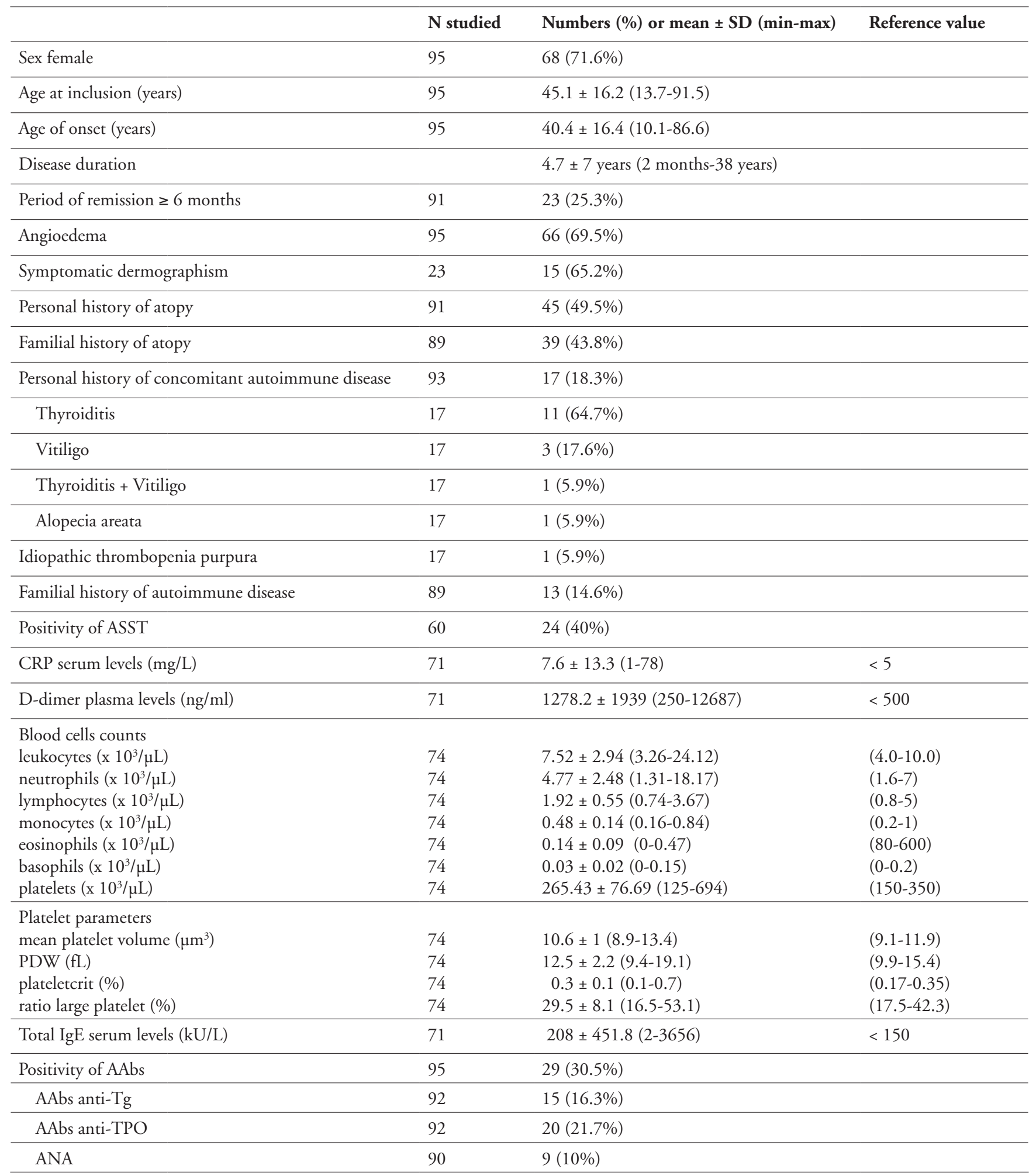


Table I - Clinical and biological data of the cohort of patients with CSU.

\begin{tabular}{|c|c|c|c|}
\hline & N studied & Numbers $(\%)$ or mean \pm SD $(\min -\max )$ & Reference value \\
\hline Autoimmune status & 94 & $32(34.0 \%)$ & \\
\hline \multirow[t]{2}{*}{$\mathrm{H}_{1}$-antihistamines } & 95 & $56(58.9 \%)$ & \\
\hline & 95 & $39(41.1 \%)$ & \\
\hline UAS7 & 59 & $21.0 \pm 12.6(0-42)$ & \\
\hline 28-42: severe & 59 & $24(40.7 \%)$ & \\
\hline 16-27: moderate & 59 & $10(16.9 \%)$ & \\
\hline 7-15: mild & 59 & $17(28.8 \%)$ & \\
\hline
\end{tabular}

Autoimmune status: concomitant autoimmune disease and/or AAbs.

$\mathrm{H}_{1}$-antihistamines response: $\mathrm{H}_{1}$-antihistamines responders (UAS7 7 with 1 to 4 tablets daily of $\mathrm{H}_{1}$-antihistamines); $\mathrm{H}_{1}$-antihistamines unresponders (UAS7 > 7 with 4 tablets daily of $\mathrm{H}_{1}$-antihistamines).

Weekly Urticaria Activity Score (UAS7) was recorded by patient for seven consecutive days prior to sampling day. Patients were classified according UAS7 as follows: severe CSU (UAS7 = 28-42), moderate CSU (UAS7 = 16-27), mild CSU (UAS7 = 7-15), well-controlled CSU (UAS7 = 1-6) and itch-and wheals-free $($ UAS7 = 0). Blood analyses that can be influenced by treatment (blood cells, platelet parameters, D-dimer plasma level, IgE and CRP serum levels, complement components) were recorded for untreated patient.

Titers for AAbs were considered positive if anti-Tg > $115 \mathrm{U} / \mathrm{ml}$, anti-TPO > $34 \mathrm{U} / \mathrm{ml}$, ANA > 1:160, and RF > 1:40. Cut-off for CRP serum level, for D-dimer plasma level and for total $\mathrm{IgE}$ serum level detection were respectively $1 \mathrm{mg} / \mathrm{L}, 250 \mathrm{ng} / \mathrm{mL}$ and $2 \mathrm{kU} / \mathrm{L}$.

Conversely, in $\mathrm{H}_{1}$-antihistamines unresponders, CRP serum levels $(\mathrm{p}<0.0001)$ (figure $1 \mathbf{A})$ and D-dimer plasma levels $(\mathrm{p}=$ 0.009) (figure $1 \mathbf{B}$ ) were significantly higher than in $\mathrm{H}_{1}$-antihistamines responders. Moreover, CRP serum levels and D-dimer plasma levels were positively correlated $(\mathrm{p}<0.0001)$.

Disease activity (based on UAS7) was positively correlated to CRP serum levels $(\mathrm{p}=0.033)$ (figure $1 \mathrm{C}$ ) and negatively correlated to blood basophil counts $(\mathrm{p}=0.023)$ (figure $1 \mathrm{D}$ ). Correlation between D-dimer plasma levels and UAS7 did not reach significance $(\mathrm{p}=0.069)$.

Disease activity was not associated with clinical parameters nor with the rest of $\mathrm{CBC}$ values, total $\operatorname{IgE}$ serum level nor with platelet parameters.

Correlation between autoimmune or autoreactive factors and biological parameters

An association between positive ASST results and autoimmune status (defined as the presence of concomitant autoimmune disease and/or AAbs) was observed ( $\mathrm{p}=0.037)$.
Positivity of ASST was correlated with angioedema $(\mathrm{p}=0.005)$ as $87.5 \%(21 / 24)$ of patients with positive ASST had angioedema in contrast to $52.8 \%$ (19/36) with negative ASST.

Blood basophil counts (figure 2 A), blood monocyte counts, and mean total IgE serum levels (figure $\mathbf{2}$ B) were lower in patients with positive ASST (respectively $\mathrm{p}=0.001, \mathrm{p}=0.019$ and $\mathrm{p}=0.016)$. However, other clinical and biological parameters were not correlated with ASST results.

$\mathrm{D}$-dimer plasma levels were higher $(\mathrm{p}=0.016)$ (figure $2 \mathrm{C}$ ), and blood basophil counts lower $(\mathrm{p}=0.057)($ figure $2 \mathrm{D})$ when an autoimmune status was present. Blood platelet counts and plateletcrit were also higher in patients with autoimmune status (respectively $\mathrm{p}=0.010$ and $\mathrm{p}=0.002$ ). Other clinical and biological parameters were not correlated with autoimmune status.

\section{Omalizumab: before initiation and under treatment}

For 22 patients, biological parameters were compared before initiation and under omalizumab. 11 patients were complete responders, 8 partial responders and 3 non-responders to omalizumab. Among responders, 17 patients were early responders 
Figure 1 - (A) Positive correlation between CRP serum levels and $H_{1}$-antihistamines response. Responders: 4.0-5.7 mg/L (1-29); unresponders: 14.7-19.9 mg/L (1-78). (B) Positive correlation between D-dimer plasma levels and $H_{1}$-antihistamines response. Responders: 1144-2093 ng/mL (250-12687); unresponders: 1558.5-1574.3 ng/mL (250-6260). (C) Positive correlation between CRP serum levels and disease activity (UAS7). (D) Negative correlation between blood basophil counts and disease activity (UAS7). Untreated patients. UAS7 recorded seven consecutive days before the blood sample.
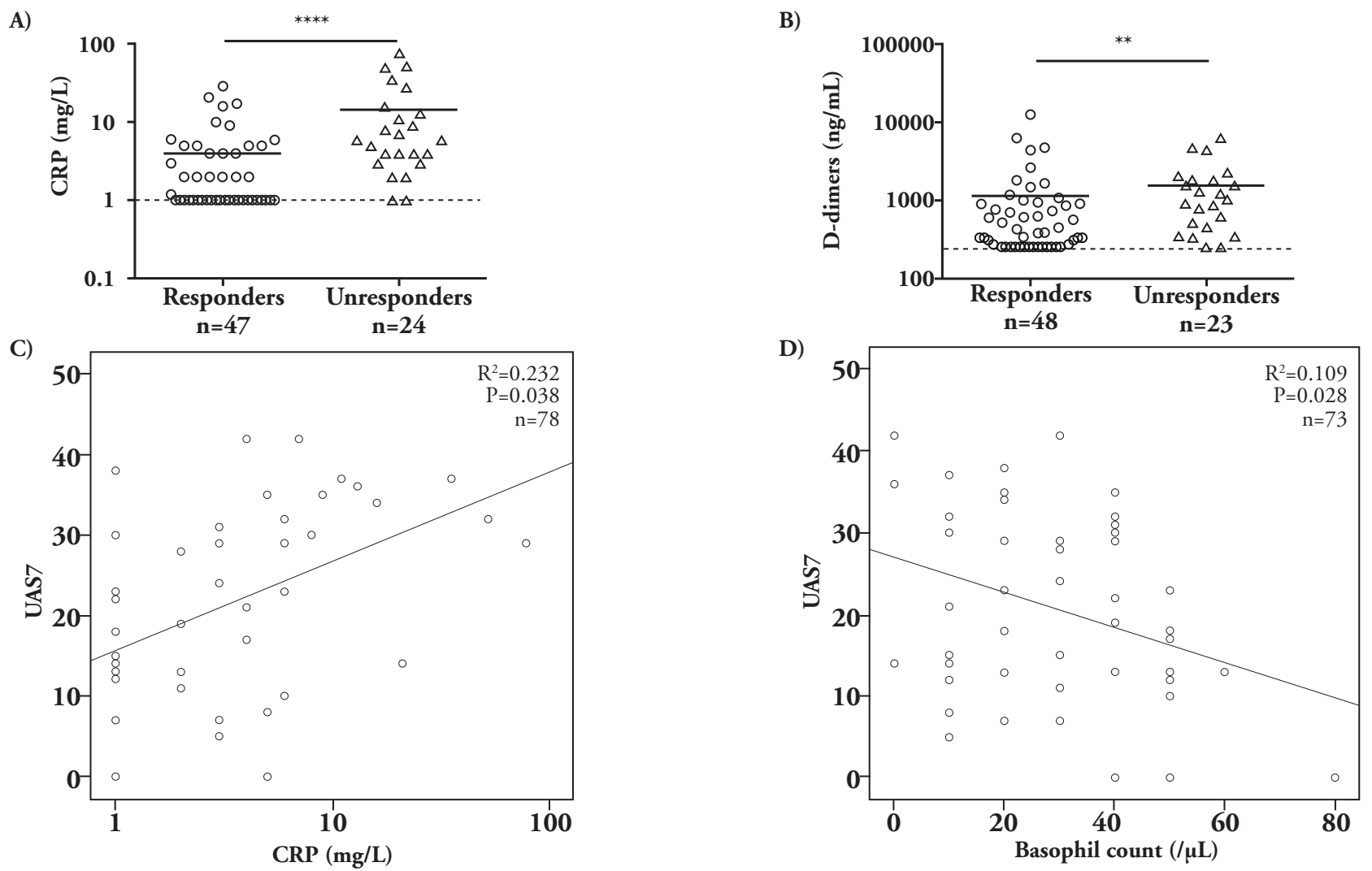

Each symbol represents one patient. Solid horizontal line represents mean.

P-value: ${ }^{*} \mathrm{p} \leq 0.05,{ }^{* *} \mathrm{p} \leq 0.01,{ }^{* * *} \mathrm{p} \leq 0.001,{ }^{* * * *} \mathrm{p} \leq 0.0001$, blank $\mathrm{p}>0.05$.

Cut-off for CRP serum level and for D-dimer plasma level detection were respectively $1 \mathrm{mg} / \mathrm{L}$ and $250 \mathrm{ng} / \mathrm{mL}$ (dotted lines).

and 2 were late ones. All data concerning patients before initiation and under omalizumab treatment are reported in table II. Significant reductions in CRP serum levels $(\mathrm{p}=0.0038)$ and D-dimer plasma levels ( $\mathrm{p}=0.0002$ ) were observed under omalizumab treatment, whereas increases were observed in blood basophil counts ( $p=0.0023$ ) (figure 3) and total IgE serum levels $(\mathrm{p}=0.0007)$. Blood basophil counts increased after omalizumab in $13 / 19$ patients, with a mean increase of $113 \%$ (20-200\%). No differences for the rest of CBC and platelet parameters were observed.

Neither clinical, nor biological parameters (including ratio of these parameters under omalizumab and before initiation) were associated with omalizumab response or with delay of response.
However, the number of patients in each group was insufficient to have a statistically significant analysis.

\section{Discussion}

Since nearly 30 years, several lines of evidence argue for an autoimmune basis of CSU, or at least in a subgroup of them. However, the way to distinguish autoimmune and non-autoimmune CSU is still a matter of debate $(13,14)$. In this study, we focused on correlations between several biological parameters, concomitant autoimmune disease and/or presence of AAbs (included IgG anti-Tg, IgG-TPO, ANA and RF) and positivity of ASST. We found a relatively high incidence of autoimmune disorders and AAbs (autoimmune status) in patients with CSU. One 
Figure 2 - (A) Lower blood basophil counts in patients with ASST + than with ASST -. ASST +: 19.6-20.6/uL (0-70); ASST-: 39.726.6/uL (10-150). (B) Lower total serum IgE levels in patients with ASST + than ASST -. ASST +: 103.4-120.2 kU/L (2-368); ASST -: 207.3-225.5 kU/L (10-1216). (C) Higher D-dimer plasma levels in patients with autoimmunity than patients without autoimmunity. AI: 2050-2952 ng/ml (256-12687); nonAI: 953. 9-1205 $\mathrm{ng} / \mathrm{ml}$ (250-6207). (D) Lower blood basophil counts in patients with autoimmunity

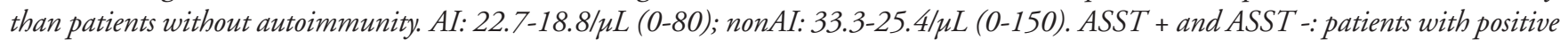
or negative ASST respectively. AI and noAI: patients with autoimmune status (concomitant autoimmune disease and/or positive for at least one AAbs) and patients without autoimmune status (no concomitant autoimmune disease, no AAbs).

A)

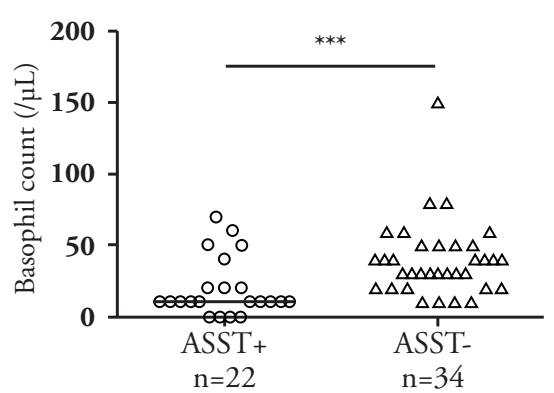

C)

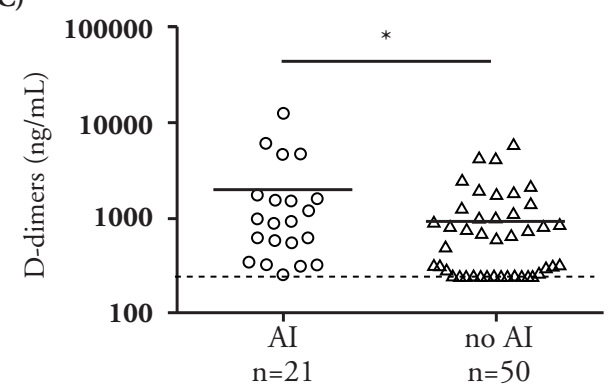

B)

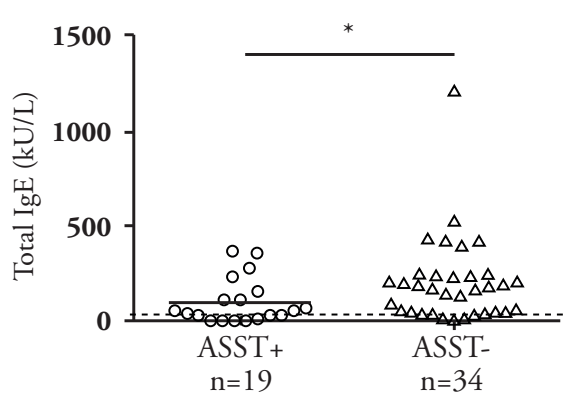

D)

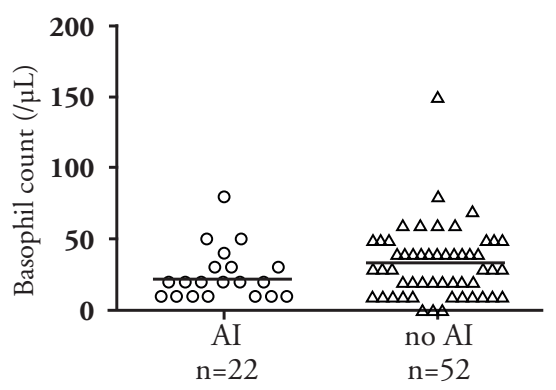

Each symbol represents one patient. Solid horizontal line represents mean.

P-value: ${ }^{*} \mathrm{p} \leq 0.05,{ }^{* *} \mathrm{p} \leq 0.01,{ }^{* * *} \mathrm{p} \leq 0.001,{ }^{* * * *} \mathrm{p} \leq 0.0001$, blank $\mathrm{p}>0.05$.

Cut-off for total IgE serum level and D-dimer plasma level detection were respectively $2 \mathrm{kU} / \mathrm{L}$ and $250 \mathrm{ng} / \mathrm{mL}$ (dotted lines).

third of the patients had concomitant autoimmune disease and/ or AAbs, mainly autoimmune thyroiditis and IgG against TPO. Moreover, a familial history of autoimmune disease was also found in $14,6 \%$ of patients. Recently, Schoepke et al. showed that autoimmune CSU (defined by the presence of $\operatorname{IgG}$ anti-IgE or anti-FceRI, a positive BAT and a positive ASST) have significantly higher IgG against TPO than patients with non-autoimmune CSU (15). In our cohort, we found a correlation between positive ASST and presence of concomitant autoimmune disease and/or AAbs. This association was not always found in previous studies, a discrepancy which could be explained by the fact that we got interest for both; presence of concomitant autoimmune disease and AAbs (16-19). The proportion of positive ASST in our cohort of patients with CSU (40\%) is consis- tent with previous reports $(30 \%$ to $50 \%)(8,20)$. Presence of concomitant autoimmune disease and/or AAbs or positivity of ASST was not correlated with disease activity nor with $\mathrm{H}_{1}$-antihistamines response. In the literature, this association between ASST and disease activity remains controversial (16, 20-22). In line with previous reports, angioedema was more frequent in patients with positive ASST in our cohort $(17,18)$. This study put forward that personal and familial autoimmune disease history as well as autoimmune serology, especially IgG against TPO, are easy to get and could be interesting to record in patients with CSU.

As several studies had postulated that CRP and IgE serum levels or D-dimer plasma level could be considered as biomarkers of CSU or CSU activity, we have measured them and looked for 
Table II - Clinical and biological data for patients treated with omalizumab. Comparison before initiation and under omalizumab.

\begin{tabular}{|c|c|c|c|c|c|}
\hline \multicolumn{6}{|c|}{ All omalizumab patients } \\
\hline & \multicolumn{2}{|r|}{ N studied } & \multicolumn{3}{|c|}{ Number $(\%)$ or mean $\pm S D(\min -\max )$} \\
\hline Sex female & \multicolumn{2}{|r|}{22} & \multicolumn{2}{|c|}{$17(77.3 \%)$} & \\
\hline Age (years) & \multicolumn{2}{|r|}{22} & \multicolumn{2}{|c|}{$42.7 \pm 14.2(13.7-70.2)$} & \\
\hline Weight (kilogrammes) & \multicolumn{2}{|r|}{4} & \multicolumn{2}{|c|}{$77.3 \pm 23.8(61-112)$} & \\
\hline Angioedema & \multicolumn{2}{|r|}{22} & \multicolumn{2}{|c|}{$16(72.7 \%)$} & \\
\hline Duration of disease (years) & \multicolumn{2}{|r|}{22} & \multicolumn{2}{|c|}{$4.5 \pm 7.9(0.5-38.3)$} & \\
\hline Period of remission $\geq 6$ months & \multicolumn{2}{|r|}{22} & \multicolumn{2}{|c|}{$2(9.1 \%)$} & \\
\hline Autoimmune status & \multicolumn{2}{|r|}{22} & \multicolumn{2}{|r|}{$9(40.9 \%)$} & \\
\hline \multirow[t]{3}{*}{ Positivity of ASST } & \multicolumn{2}{|r|}{10} & \multicolumn{2}{|r|}{$4(40 \%)$} & \\
\hline & \multicolumn{2}{|c|}{ Before omalizumab initiation } & \multicolumn{2}{|c|}{ Under omalizumab } & \\
\hline & N studied & $\begin{array}{c}\text { number }(\%) \text { or mean } \pm \\
\text { SD (min-max) }\end{array}$ & N studied & $\begin{array}{c}\text { number }(\%) \text { or mean } \pm \text { SD } \\
(\text { min-max })\end{array}$ & P-value \\
\hline UAS7 & 18 & $31.4 \pm 7.4(17-42)$ & 12 & $6.3 \pm 11.4(0-38)$ & \\
\hline \multicolumn{6}{|l|}{ Activity based on UAS7 } \\
\hline 28-42: severe & 18 & 14 & 12 & 1 & \\
\hline 16-27: moderate & 18 & 4 & 12 & 1 & \\
\hline 7-15: mild & 18 & 0 & 12 & 2 & \\
\hline 1-6: well-controlled & 18 & 0 & 12 & 2 & \\
\hline $0:$ itch-and wheals-free & 18 & 0 & 12 & 6 & \\
\hline CRP serum levels (mg/L) & 18 & $18 \pm 21.9(1-78)$ & 18 & $5.1 \pm 5.4(1-23)$ & 0.0038 \\
\hline D-dimer plasma levels (ng/ml) & 17 & $1668 \pm 1795(250-6260)$ & 17 & $397.1 \pm 307.8(250-1248)$ & 0.0002 \\
\hline Leukocytes $\left(\right.$ x $\left.10^{3} / \mu \mathrm{L}\right)$ & 19 & $7.9 \pm 2.4(4.7-12.2)$ & 19 & $7.4 \pm 2.1(4.3-12)$ & 0.35 \\
\hline 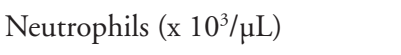 & 19 & $5.2 \pm 2.1(2.4-9.1)$ & 19 & $4.6 \pm 1.6(2.4-8.2)$ & 0.10 \\
\hline 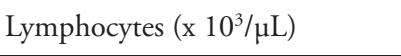 & 19 & $2.1 \pm 0.7(0.7-3.7)$ & 19 & $2.1 \pm 0.7(0.6-3.8)$ & \\
\hline Monocytes $\left(\mathrm{x} 10^{3} / \mu \mathrm{L}\right)$ & 19 & $0.5 \pm 0.2(0.2-0.7)$ & 19 & $0.5 \pm 1.1(0.3-0.7)$ & 0.25 \\
\hline Eosinophils $\left(\mathrm{x} 10^{3} / \mu \mathrm{L}\right)$ & 19 & $0.1 \pm 0.08(0-0.3)$ & 19 & $0.1 \pm 0.06(0-0.2)$ & 0.83 \\
\hline Basophils (x $\left.10^{3} / \mu \mathrm{L}\right)$ & 19 & $0.02 \pm 0.01(0-0.05)$ & 19 & $0.03 \pm 0.02(0-0.08)$ & 0.0023 \\
\hline Platelets $\left(\mathrm{x} 10^{3} / \mu \mathrm{L}\right)$ & 19 & $282 \pm 58.2(196-378)$ & 19 & $275.7 \pm 39.3(216-359)$ & 0.75 \\
\hline Mean platelet volume $\left(\mu \mathrm{m}^{3}\right)$ & 19 & $10.5 \pm 0.9(9.1-12.6)$ & 19 & $10.7 \pm 0.8(9.1-12)$ & 0.15 \\
\hline Total IgE serum levels (kU/L) & 18 & $137.4 \pm 121.9(2-425)$ & 18 & $458.6 \pm 420.7(2-1243)$ & 0.0007 \\
\hline \multicolumn{6}{|l|}{ Response to omalizumab } \\
\hline complete responders & & & 22 & $11(50 \%)$ & \\
\hline partial responders & & & 22 & $8(36.4 \%)$ & \\
\hline non responders & & & 22 & $3(13.6 \%)$ & \\
\hline \multicolumn{6}{|l|}{ Time to respond to omalizumab } \\
\hline early responders & & & 19 & $17(89.5 \%)$ & \\
\hline late responders & & & 19 & $2(10.5 \%)$ & \\
\hline
\end{tabular}

Autoimmune status: concomitant autoimmune disease and/or AAbs.

Weekly Urticaria Activity Score (UAS7) was recorded by patient for seven consecutive days prior to sampling day.

Titers for AAbs were considered positive if anti-Tg > $115 \mathrm{U} / \mathrm{ml}$, anti-TPO $>34 \mathrm{U} / \mathrm{ml}$, ANA > 1:160, and RF > 1:40. Cut-off for CRP serum level, for D-dimer plasma level and for total IgE serum level detection were respectively $1 \mathrm{mg} / \mathrm{L}, 250 \mathrm{ng} / \mathrm{mL}$ and $2 \mathrm{kU} / \mathrm{L}$. 
Figure 3 - Elevation of blood basophil counts under omalizumab treatment. Before omalizumab initiation: $20.5 \pm 13.5 / \mu \mathrm{L}(0-50)$; under omalizumab: $31.6 \pm 18.6 / \mu L(0-80)$.

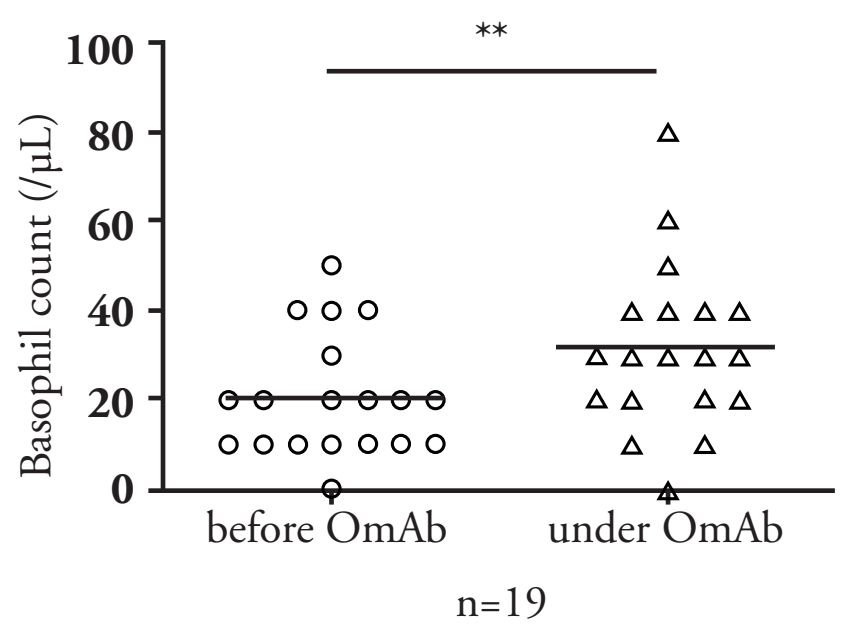

Each symbol represents one patient. Solid horizontal line represents mean. P-value: ${ }^{*} \mathrm{p} \leq 0.05,{ }^{* *} \mathrm{p} \leq 0.01,{ }^{* * *} \mathrm{p} \leq 0.001,{ }^{* * *} \mathrm{p} \leq 0.0001$, blank $\mathrm{p}>0.05$. Before OmAb: patients before beginning of omalizumab treatment; under OmAb: patients under omalizumab.

correlations with each other, and with clinical data in this prospective study of 95 CSU patients.

CRP serum level has been proposed as a biomarker of disease activity in patients with CSU, however results are contradictory $(23,24$.) Our findings show that CRP (mean CRP 7.7 $\mathrm{mg} / \mathrm{L}$ ) was higher in CSU patients with more active disease and in $\mathrm{H}_{1}$-antihistamines unresponders. However, to our opinion, CRP is not an interesting biomarker in CSU due to its relatively low levels compared with others inflammatory diseases and due to possible fluctuations by intercurrent phenomena which are not always reported especially in retrospective studies. CRP is more useful in the differential diagnoses of CSU, a high CRP level, not reducing under treatment, could be a sign of an auto-inflammatory syndrome.

Several studies have shown 1) higher D-dimer plasma levels in patients with CSU compared with HCs, 2) higher D-dimer plasma levels in more active disease, as well as 3) normalization of D-dimer plasma levels during remission $(25,26)$. In our cohort, D-dimer plasma levels were higher in $\mathrm{H}_{1}$-antihistamines unresponders and to a lesser extent, in more active disease. Interestingly, we also found that patients with autoimmune status had higher D-dimer plasma levels than those without autoimmunity. Furthermore, in line with previous reports, we found a significant decrease of D-dimer plasma levels under omalizum$\mathrm{ab}$ treatment. Baseline D-dimer plasma levels do not predict the response to omalizumab (27-29), whereas the decrease of
D-dimer plasma levels under omalizumab treatment seem to be a marker of good response to treatment (30). Measurement of D-dimer plasma levels could be added to clinical tools, such as UAS7, to evaluate activity/severity. Concerning response to treatment, as well as relation between D-dimer plasma levels and autoimmunity, further studies are needed.

Mean total IgE serum levels have often been studied in patients with CSU and have been proposed as a biomarker of disease activity, however, results remain contradictory $(31,32)$. In our study, the mean total IgE serum levels of untreated patients were in normal range or little high $(208.2 \pm 451.8 \mathrm{kU} / \mathrm{L}$, except in atopic patients for which is high), and less than half of patients had total $\mathrm{IgE}$ serum levels higher than normal reference value. Total IgE serum levels didn't correlate with disease activity nor with response to $\mathrm{H}_{1}$-antihistamines. Interestingly total IgE serum levels were lower in patients with positive ASST (mean 103.37 $\pm 120.25 \mathrm{kU} / \mathrm{L}$ ) compared with negative ASST (mean $207.32 \pm$ $225.51 \mathrm{kU} / \mathrm{L})(\mathrm{p}=0.016)$. This finding is in line with recent publications showing that very low total IgE and positive ASST have been relat ed with poor/no response and with slow response to omalizumab respectively $(27,33-36)$. Moreover, Schoepke et al. put forward that autoimmune CSU have significantly lower total IgE serum levels than non-autoimmune CSU (15). Low baseline IgE has been described as a marker of poor response $(27,34,37)$. However this has not been confirmed by all studies $(38,39)$. Interestingly, a recent paper suggest that total IgE levels can be used as predictors of response to omalizumab only in nonatopic CSU patients, actually they showed that the atopic status modify the ability of IgE to predict the response to the treatment (40).

Several authors have discussed a possible main role of basophils. Indeed, in our study, we found that blood basophil counts were significantly lower in patients with CSU compared with healthy controls. Moreover, blood basophil counts correlated with disease activity. Basophils are probably recruited into the skin during wheal formation, as evidenced by an abundance of basophils in skin samples $(41,42)$, and low blood basophil counts in patients with chronic urticaria $(43,44)$. Furthermore, in our study, blood basophil counts were significantly lower in patients with positive ASST, and also tended to be correlated with presence of concomitant autoimmune disease and/or AAbs. This correlation has previously been poorly investigated and with controversial results $(21,43,45,46)$. Nevertheless, expression of activation markers, such as CD203c and CD63, has been found to be higher in blood basophils of CSU patients with positive ASST compared to patients with negative ASST $(45,47)$. It is tempting to speculate that in patients with positive ASST, basophils are implicated and activated in a more important way, and thus reduced in blood due to recruitment into the skin. Mechanisms implicated in basophils activation/recruitment in skin are actually unknown, however according to previous findings, AAbs could be indirectly implicated. 
Additionally, under omalizumab treatment, we observed a significant increase in blood basophil counts, suggesting that omalizumab blocks this basophil shift from the bloodstream into the skin. Unfortunately, our cohort of patients was too small to identify differences between responders/unresponders and fast/ slow responders to omalizumab. In the same line, previous authors have already observed increased blood basophil counts in correlation with improvement on treatment or remission (48, 49). However, data concerning blood basophil counts in patients with CSU under omalizumab treatment are scarce (50-53).

Consistence of this study is to have analyzed several biomarkers, evaluated in untreated patients, as well as a series of clinical parameters in a prospective cohort. Limitations are mainly due to the fact that correlations between clinical/biological parameters and omalizumab response (or delay of response) were not possible due to the small number of patients in each group. Secondly, concerning autoimmune CSU investigations, we haven't performed functional tests as BAT and BHRA nor IgG anti FceRI/IgE measurement.

\section{Conclusions}

To conclude, in this prospective study, we found a relatively high incidence of concomitant autoimmune disease and AAbs. D-dimer plasma level is higher in $\mathrm{H}_{1}$-antihistamines unresponders and in patients with autoimmune status. Total $\mathrm{IgE}$ serum levels were lower in patients with positive ASST compared with negative ASST. We found lower blood basophil counts in patients with CSU compared with healthy controls. Moreover, this finding was more significant in patients with positive ASST and to a lesser extent in patients with autoimmune status. Moreover, under omalizumab, blood basophil counts and total IgE serum levels increased and conversely D-dimer plasma levels decrease. Our study brings additional evidences over the utility of those clinical and biological parameters to investigate in patients with $\mathrm{CSU}$ as they could be related to disease activity, response to treatment or autoimmunity.

\section{Acknowledgments}

We thank the Société Française de Dermatologie for the grant accorded to Dr Anne-Sophie Darrigade. We thank Dr JeanPhilippe Defour and Dr Charlotte Fauconnier for their helpful involvement. We thank Dr Mariana Andrade who provided editorial assistance. Dr Andrade received payment for her services but no commercial funding was received for this purpose.

\section{Conflict of interests}

L.d.M. reports medical investigator/advisor and educational activities for Novartis. A-S.D. reports educational activities for Novartis. A.G.A. acted as medical advisor for Uriach Pharma, Genentech, Novartis, FAES, GSK, Sanofi. A.G.A reports re- search Grants supported by Uriach Pharma, Novartis, Grants from Instituto Carlos III- FEDER and educational activities for Uriach Pharma, Novartis, Genentech, Menarini, LEO- PHARMA, GSK, MSD, Almirall, and Sanofi. M.B. reports medical investigator/advisor and educational activities for Novartis.

\section{References}

1. Confino-Cohen R, Chodick G, Shalev V, Leshno M, Kimhi O, Goldberg A. Chronic urticaria and autoimmunity: Associations found in a large population study. J Allergy Clin Immunol 2012;129(5):1307-13.

2. Chiu HY, Muo CH, Sung FC. Associations of chronic urticaria with atopic and autoimmune comorbidities: a nationwide population-based study. Int J Dermatol 2018;57(7):822-9.

3. Pan X-F, Gu J-Q, Shan Z-Y. The prevalence of thyroid autoimmunity in patients with urticaria: a systematic review and meta-analysis. Endocrine 2014;48(3):804-10.

4. Lee M-F, Lin T-M, Liu S-W, Chen Y-H. A Rapid Method of Detecting Autoantibody against FceRI $\alpha$ for Chronic Spontaneous Urticaria. PLoS ONE 2014;9(10):e109565.

5. Tong LJ, Balakrishnan G, Kochan JP, Kinet JP, Kaplan AP. Assessment of autoimmunity in patients with chronic urticaria. J Allergy Clin Immunol 1997;99(4):461-5.

6. Schmetzer O, Lakin E, Topal FA, et al. IL-24 is a common and specific autoantigen of $\mathrm{IgE}$ in chronic spontaneous urticaria. J Allergy Clin Immunol 2018;142(3):876-82.

7. Altrichter S, Peter H-J, Pisarevskaja D, Metz M, Martus P, Maurer M. IgE Mediated Autoallergy against Thyroid Peroxidase - A Novel Pathomechanism of Chronic Spontaneous Urticaria? PLoS ONE 2011;6(4):e14794.

8. Konstantinou GN, Asero R, Maurer M, Sabroe RA, Schmid-Grendelmeier P, Grattan CEH. EAACI/GA2LEN task force consensus report: the autologous serum skin test in urticaria. Allergy 2009;64(9):1256-68.

9. Yasnowsky KM, Dreskin SC, Efaw B, et al. Chronic urticaria sera increase basophil CD203c expression. J Allergy Clin Immunol 2006;117(6):1430-4.

10. Zuberbier T, Aberer W, Asero R, et al. The EAACI/GA(2) LEN/ EDF/WAO Guideline for the definition, classification, diagnosis, and management of urticaria: the 2013 revision and update. Allergy 2014;69(7):868-87.

11. Khalil S, McBride D, Gimenez-Arnau A, Grattan C, Balp M, Stull D. Weekly Urticaria Activity Score (UAS7) and Dermatology Life Quality Index (DLQI) in Validation of Chronic Spontaneous/ Idiopathic Urticaria (CSU/CIU) Health States. Annual Scientific Meeting of the American Academy of Allergy, Asthma \& Immunology; Houston, Texas, USA 2015.

12. Zuberbier T, Aberer W, Asero R, et al. The EAACI/GA(2)LEN/ EDF/WAO Guideline for the Definition, Classification, Diagnosis and Management of Urticaria. The 2017 Revision and Update. Allergy 2018;73(7):1393-414

13. Kolkhir P, Church MK, Weller K, Metz M, Schmetzer O, Maurer M. Autoimmune chronic spontaneous urticaria: What we know and what we do not know. J Allergy Clin Immunol 2017;139(6):1772-81.e1.

14. Grattan C. Autoimmune chronic spontaneous urticaria. J Allergy Clin Immunol 2018;141(3):1165-6. 
15. Schoepke N, Asero R, Ellrich A, et al. Biomarkers and clinical characteristics of autoimmune chronic spontaneous urticaria: Results of the PURIST Study. Allergy 2019.

16. Chanprapaph K, Iamsumang W, Wattanakrai P, Vachiramon V. Thyroid Autoimmunity and Autoimmunity in Chronic Spontaneous Urticaria Linked to Disease Severity, Therapeutic Response, and Time to Remission in Patients with Chronic Spontaneous Urticaria. Biomed Res Int 2018;2018:9856843.

17. Kumar YH, Bhaskar S, Shankar K. Comparative Study of Positive Versus Negative Autologous Serum Skin Test in Chronic Spontaneous Urticaria and its Treatment Outcome. North Am J Med Sci 2016;8(1):25-30.

18. Altrich ML, Halsey JF, Altman LC. Comparison of the in vivo autologous skin test with in vitro diagnostic tests for diagnosis of chronic autoimmune urticaria. Allergy and asthma proceedings: the official journal of regional and state allergy societies 2009;30(1):28-34.

19. Magen E, Mishal J, Zeldin Y, et al. Increased mean platelet volume and C-reactive protein levels in patients with chronic urticaria with a positive autologous serum skin test. Am J Med Sci 2010;339(6):504-8.

20. Curto-Barredo L, Archilla LR, Vives GR, Pujol RM, Gimenez-Arnau AM. Clinical Features of Chronic Spontaneous Urticaria that Predict Disease Prognosis and Refractoriness to Standard Treatment. Acta Derm Venereol 2018;98(7):641-7.

21. Ye YM, Park JW, Kim SH, et al. Prognostic Factors for Chronic Spontaneous Urticaria: A 6-Month Prospective Observational Study. Allergy Asthma Immunol Res 2016;8(2):115-23.

22. Curto-Barredo L, Yelamos J, Gimeno R, Mojal S, Pujol RM, Gimenez-Arnau A. Basophil Activation Test identifies the patients with Chronic Spontaneous Urticaria suffering the most active disease. Immun Inflamm Dis 2016;4(4):441-5.

23. Kolkhir P, Altrichter S, Hawro T, Maurer M. C-reactive protein is linked to disease activity, impact, and response to treatment in patients with chronic spontaneous urticaria. Allergy 2018;73(4):940-8.

24. Baek YS, Jeon J, Kim JH, Oh CH. Severity of acute and chronic urticaria correlates with D-dimer level, but not C-reactive protein or total IgE. Clin Exp Dermatol 2014;39(7):795-800.

25. Wang F, Tang H, Xu JH, Kang KF. Activation of the blood coagulation cascade is involved in patients with chronic urticaria. J Allergy Clin Immunol 2009;123(4):972-3; author reply 973-4.

26. Asero R, Tedeschi A, Coppola R, et al. Activation of the tissue factor pathway of blood coagulation in patients with chronic urticaria. J Allergy Clin Immunol 2007;119(3):705-10.

27. Marzano AV, Genovese G, Casazza G, et al. Predictors of response to omalizumab and relapse in chronic spontaneous urticaria: a study of 470 patients. J Eur Acad Dermatol Venereol 2018;33(5):918-24.

28. Asero R, Marzano AV, Ferrucci S, Genovese G, Cugno M. Baseline D-dimer plasma levels correlate with disease activity but not with the response to omalizumab in chronic spontaneous urticaria. Allergy 2019;74(12):2538.

29. Hamelin A, Amsler E, Mathelier-Fusade P, et al. Omalizumab for the treatment of chronic urticaria: Real-life findings. Ann Dermatol Venereol 2019;146(1):9-18.

30. Asero R, Marzano AV, Ferrucci S, Cugno M. D-Dimer Plasma Levels Parallel the Clinical Response to Omalizumab in Patients with Severe Chronic Spontaneous Urticaria. Int Arch Allergy Immunol 2017;172(1):40-4.
31. Toubi E, Kessel A, Avshovich N, et al. Clinical and laboratory parameters in predicting chronic urticaria duration: a prospective study of 139 patients. Allergy 2004;59(8):869-73.

32. Kessel A, Helou W, Bamberger E, et al. Elevated serum total IgE--a potential marker for severe chronic urticaria. Int Arch Allergy Immunol 2010;153(3):288-93.

33. Ertas R, Ozyurt K, Ozlu E, et al. Increased IgE levels are linked to faster relapse in patients with omalizumab-discontinued chronic spontaneous urticaria. J Allergy Clin Immunol 2017;140(6):1749-51.

34. Deza G, Bertolin-Colilla M, Pujol RM, et al. Basophil FcepsilonRI Expression in Chronic Spontaneous Urticaria: A Potential Immunological Predictor of Response to Omalizumab Therapy. Acta Derm Venereol 2017;97(6):698-704.

35. Weller K, Ohanyan T, Hawro T, et al. Total IgE levels are linked to the response of chronic spontaneous urticaria patients to omalizumab. Allergy 2018;73(12):2406-8.

36. Nettis E, Cegolon L, Di Leo E, Lodi Rizzini F, Detoraki A, Canonica GW. Omalizumab chronic spontaneous urticaria: Efficacy, safety, predictors of treatment outcome, and time to response. Ann Allergy Asthma Immunol 2018;121(4):474-8.

37. Ertas R, Ozyurt K, Atasoy M, Hawro T, Maurer M. The clinical response to omalizumab in CSU patients is linked to and predicted by IgE levels and their change. Allergy 2018;73:705-12.

38. Ghazanfar MN, Holm JG, Thomsen SF. Effectiveness of omalizumab in chronic spontaneous urticaria assessed with patient-reported outcomes: a prospective study. J Eur Acad Dermatol Venereol 2018;32(10):1761-7.

39. Metz M, Ohanyan T, Church MK, Maurer M. Omalizumab is an effective and rapidly acting therapy in difficult-to-treat chronic urticaria: a retrospective clinical analysis. J Dermatol Sci 2014;73(1):57-62.

40. Asero R, Ferrucci S, Casazza G, Marzano AV, Cugno M. Total $\mathrm{IgE}$ and atopic status in patients with severe chronic spontaneous urticaria unresponsive to omalizumab treatment. Allergy 2019;74(8):1561-3.

41. Caproni M, Giomi B, Volpi W, et al. Chronic idiopathic urticaria: infiltrating cells and related cytokines in autologous serum-induced wheals. Clinical immunology 2005;114(3):284-92.

42. Ying S, Kikuchi Y, Meng Q, Kay AB, Kaplan AP. TH1/TH2 cytokines and inflammatory cells in skin biopsy specimens from patients with chronic idiopathic urticaria: Comparison with the allergen-induced late-phase cutaneous reaction. J Allergy Clin Immunol 2002;109(4):694-700.

43. Magen E, Zueva E, Mishal J, Schlesinger M. The clinical and laboratory characteristics of acute spontaneous urticaria and its progression to chronic spontaneous urticaria. Allergy and asthma proceedings : the official journal of regional and state allergy societies 2016;37(5):394-9.

44. Deza G, March-Rodriguez A, Sanchez S, et al. Relevance of the basophil high-affinity IgE receptor in chronic urticaria: Clinical experience from a tertiary care institution. The journal of allergy and clinical immunology In practice 2019;7(5):1619-26.e1.

45. Lourenco FD, Azor MH, Santos JC, et al. Activated status of basophils in chronic urticaria leads to interleukin-3 hyper-responsiveness and enhancement of histamine release induced by anti-IgE stimulus. Br J Dermatol 2008;158(5):979-86.

46. Grattan CE, Walpole D, Francis DM, et al. Flow cytometric analysis of basophil numbers in chronic urticaria: basopenia is related to serum histamine releasing activity. Clin Exp Allergy 1997;27(12):1417-24. 
47. Chen Q, Zhai Z, Xu J, et al. Basophil CD63 expression in chronic spontaneous urticaria: correlation with allergic sensitization, serum autoreactivity and basophil reactivity. J Eur Acad Dermatol Venereol 2017;31(3):463-8.

48. Grattan CE, Dawn G, Gibbs S, Francis DM. Blood basophil numbers in chronic ordinary urticaria and healthy controls: diurnal variation, influence of loratadine and prednisolone and relationship to disease activity. Clin Exp Allergy 2003;33(3):337-41.

49. Oliver ET, Sterba PM, Saini SS. Interval shifts in basophil measures correlate with disease activity in chronic spontaneous urticaria. Allergy 2015;70(5):601-3.

50. Kishimoto I, Kambe N, Ly NTM, Nguyen CTH, Okamoto H. Basophil count is a sensitive marker for clinical progression in a chronic spontaneous urticaria patient treated with omalizumab. Allergology international : official journal of the Japanese Society of Allergology 2019:S1323-8930.

51. Johal K, MacGlashan D, Schroeder JT, Oliver E, Chichester K, Saini S. Kinetic Profiling of Clinical Symptoms and Basophil Parameters During Treatment of Chronic Spontaneous Urticaria with Omalizumab. J Allergy Clin Immunol 2019;143(2):AB49.

52. Saini SS, Omachi TA, Trzaskoma B, et al. Effect of Omalizumab on Blood Basophil Counts in Patients with Chronic Idiopathic/ Spontaneous Urticaria. J Invest Dermatol 2017;137(4):958-61.

53. Alizadeh Aghdam M, Knol EF, van den Elzen M, et al. Response of FcepsilonRI-bearing leukocytes to omalizumab in chronic spontaneous urticaria. Clin Exp Allergy 2020;50(3):364-371. 